\title{
Bias dependence of spin injection into GaAs from Fe, FeCo, and $(\mathrm{Ga}, \mathrm{Mn})$ As contacts
}

\author{
B. Endres, ${ }^{\text {a) }}$ F. Hoffmann, C. Wolf, A. Einwanger, M. Utz, D. Schuh, G. Woltersdorf, \\ M. Ciorga, D. Weiss, C.H. Back, and G. Bayreuther \\ Institut für Experimentelle und Angewandte Physik, Universität Regensburg, 93040 Regensburg, Germany
}

(Presented 15 November 2010; received 20 October 2010; accepted 9 November 2010; published online 22 March 2011)

\begin{abstract}
Spin injection from $\mathrm{Fe}(001)$ and $(\mathrm{Ga}, \mathrm{Mn}) \mathrm{As}(001)$ into $\mathrm{n}-\mathrm{GaAs}(001)$ was investigated using a method which provides two-dimensional cross-sectional images of the spin polarization in GaAs. While the distribution of the spin polarization below the injecting contact is nearly uniform for $(\mathrm{Ga}, \mathrm{Mn}) \mathrm{As}$, a strong confinement near the contact edge is observed for $\mathrm{Fe}$ and $\mathrm{FeCo}$. The spin polarization in GaAs changes sign when the injected current is reversed. Multiple sign reversals as a function of bias voltage as reported previously for Fe injectors are not observed with (Ga,Mn)As and $\mathrm{Fe}$ contacts grown on clean $n^{++}-\mathrm{GaAs}$ in agreement with earlier results for an epitaxial $\mathrm{FeCo}$ injector. @ 2011 American Institute of Physics. [doi:10.1063/1.3553932]
\end{abstract}

\section{INTRODUCTION}

Spin injection from a ferromagnetic contact into a semiconductor is a fundamental prerequisite for future spintronic devices. Majority spin injection into $\operatorname{GaAs}(001)$ has been observed from $\mathrm{Fe}^{1}$ and $\mathrm{FeCo}^{2}$ epitaxial contacts. However, a complex bias dependence of the spin polarization was found in the case of $\mathrm{Fe}$ contacts changing from sample to sample ${ }^{1}$ in contrast to FeCo which showed the expected sign reversal of spin polarization when changing from electron injection to extraction. To shed light on the effect of bias, spin injection into GaAs from $\mathrm{Fe}$ and $(\mathrm{Ga}, \mathrm{Mn}) \mathrm{As}$ was studied by a cross-sectional imaging method ${ }^{2}$ which yields the twodimensional spin polarization distribution in the semiconductor even below the contacts.

\section{METHODS}

The geometry of sample and measurement is shown in Fig. 1. All layers were grown by a molecular beam epitaxy on semi-insulating GaAs substrates. The samples with $\mathrm{Fe}$ or $\mathrm{FeCo}$ contacts consist of a $4 \mu \mathrm{m}$ thick $\mathrm{n}$-GaAs layer $\left(n=2 \cdot 10^{16} \mathrm{~cm}^{-3}\right)$, followed by a $15 \mathrm{~nm}$ thick transition layer $n \rightarrow n^{++}$and a $15 \mathrm{~nm} n^{++}-$GaAs layer. Details for the FeCo growth can be found in Ref. 2. The sample was then transferred into a second MBE chamber under UHV conditions where $\mathrm{Fe}(2 \mathrm{~nm})$ and $\mathrm{Au}(5 \mathrm{~nm})$ were deposited.

For $(\mathrm{Ga}, \mathrm{Mn})$ As contacts, the sequence of layers was as follows: $500 \mathrm{~nm}$ of a GaAs/AlGaAs superlattice, $3 \mu \mathrm{m}$ of a lightly Si-doped $\mathrm{n}$-GaAs epilayer $\left(n=4 \cdot 10^{16} \mathrm{~cm}^{-3}\right), 15$ $\mathrm{nm}$ of a $n \rightarrow n^{++}$Si-doped GaAs transition layer $\left(n^{++}\right.$ $\left.=6 \cdot 10^{18} \mathrm{~cm}^{-3}\right), 8 \mathrm{~nm}$ of $n^{++}-\mathrm{GaAs}, 2.2 \mathrm{~nm}$ of low-temperature (LT)-grown $\mathrm{Al}_{36} \mathrm{Ga}_{66} \mathrm{As}$, and $20 \mathrm{~nm}$ of a LT-grown $\mathrm{Ga}_{95} \mathrm{Mn}_{5} \mathrm{As}$. The tunneling Esaki diode structure, transforming spin polarized holes to electrons, is formed by the p-type

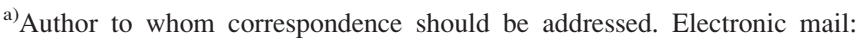
Bernhard.Endres@physik.uni-r.de.
}

(Ga,Mn)As layer and $n^{++}-$GaAs layer as described elsewhere. ${ }^{3}$

Fully epitaxial growth was verified by a RHEED in all cases. Optical lithography and Ar ion etching were used to define the contact pads. Finally, the samples were cleaved along the [1-10] direction across the ferromagnetic pads, thus exposing the (110) surface and enabling direct optical access to the n-GaAs channel (see Fig. 1). For the optical measurements the sample was mounted in a He flow cryostat. The cryostat itself is mounted on top of a nano positioner, thus two-dimensional scans can be performed by moving the sample under the static laser beam. The $\mathrm{z}$ component of the electron spin polarization (i.e., the component along [110]) in the $\mathrm{n}$-GaAs channel is detected via the polar magneto-optical Kerr effect (pMOKE). The photon energy of the linearly polarized laser beam was chosen slightly below the bandgap of $\operatorname{GaAs}(\lambda=819 \mathrm{~nm}$ at $10 \mathrm{~K})$; here, the specific Kerr rotation shows a maximum and the penetration depth of the light of more than $2 \mu \mathrm{m}$ is significantly larger than the depletion depth of the GaAs. A square-wave bias voltage alternating between zero and $V_{B}$ is applied between two ferromagnetic contacts and the Kerr rotation is detected synchronously with balanced photo-receivers and a lock-in technique. This ensures that the (quasistatic) magnetization of the ferromagnetic contacts does not contribute to the Kerr signal.

The Kerr rotation angle, $\theta_{K}$, versus the magnetic field applied along the [110] direction (z direction) at a distance of $1 \mu \mathrm{m}$ below the contact is shown in Fig. 1. The Kerr signal exactly reproduces the magnetization curve of the (Ga,Mn)As or Fe injector, resp., which clearly demonstrates that spins are indeed injected from the ferromagnetic contact into the semiconductor. The same behavior was observed for FeCo before. ${ }^{2}$

\section{RESULTS}

Figure 2 shows two-dimensional scans of the Kerr rotation in GaAs, illustrated as a color coded map for the 

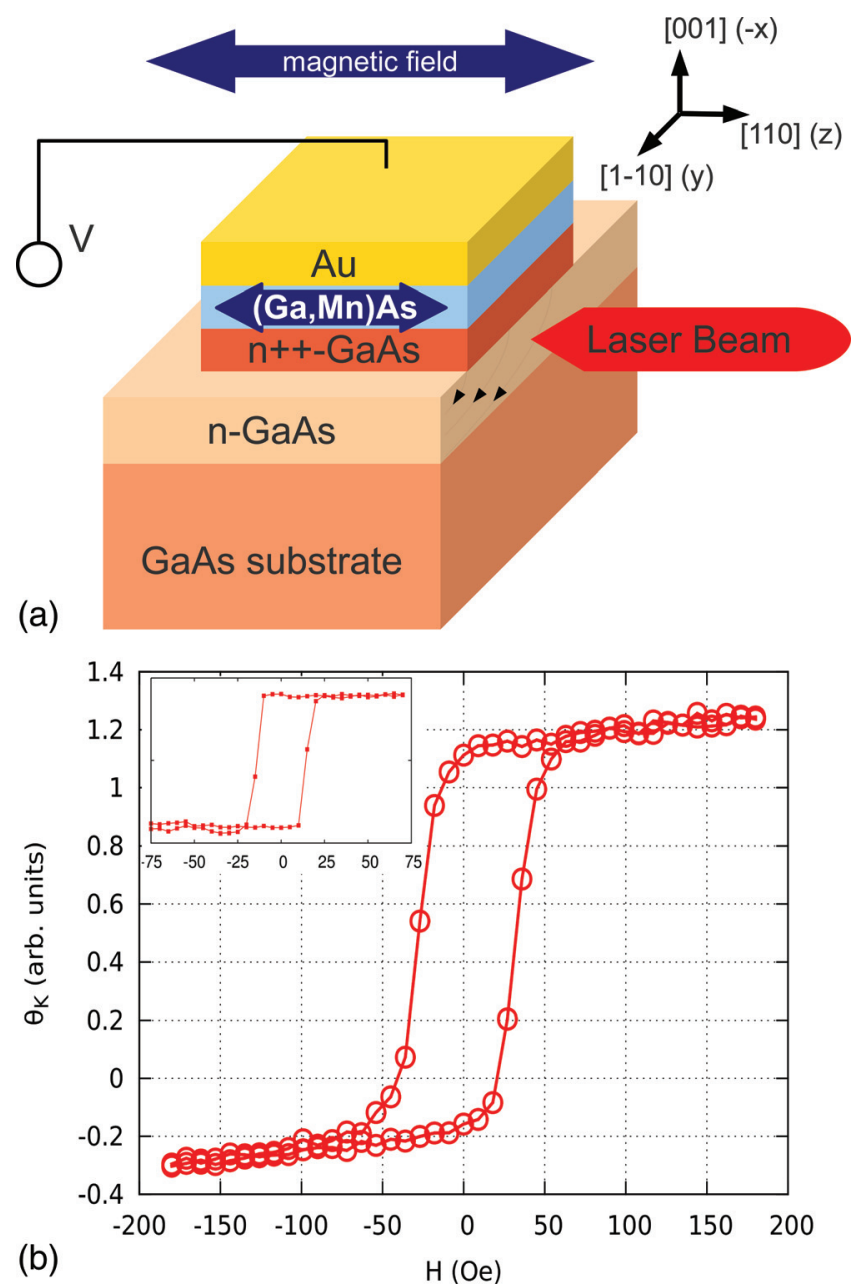

FIG. 1. (Color online) (a) Basic geometry of the sample and measurement principle. Voltage $\mathrm{V}$ is applied between two neighboring contacts. (b) pMOKE Kerr rotation in the $\mathrm{n}$-GaAs channel vs. applied magnetic field below the $(\mathrm{Ga}, \mathrm{Mn})$ As contact representing the spin polarization in the semiconductor(inset shows respective signal for an Fe contact).

injection and the extraction case from $(\mathrm{Ga}, \mathrm{Mn})$ As. The measurement was done in remanence after saturation along [110] and $[-1-10]$, respectively and the difference between both remanent values is used as a measure of the spin polarization in GaAs. The decay of the spin polarization in both directions away from the $(\mathrm{Ga}, \mathrm{Mn}) \mathrm{As}$ contact can be well fitted with an exponential. While the decay to the left is a superposition of drift and diffusion and depends on the applied bias (9.1 $\mu \mathrm{m}$ decay length for injection), the spin diffusion length can be extracted from the exponential decay to the right and resulted to $3.3 \pm 0.3 \mu \mathrm{m}$ for this sample.

Figure 3 shows two-dimensional scans with $\mathrm{Fe}$ as an injector, again for both spin injection and extraction. A significant difference is observed in the spin density distribution for both contact materials. While the spin polarization for the $(\mathrm{Ga}, \mathrm{Mn}) \mathrm{As}$ sample shows only slight variations below the contact for injection and extraction, the spin injection from $\mathrm{Fe}$ is mainly concentrated at the edge of the contact. A similar behavior was seen earlier for the $\mathrm{FeCo}$ contact. ${ }^{2}$ Interestingly, the spin extraction is even more confined to the Fe contact edge and the spin polarization decays to a negligible value at about $10 \mu \mathrm{m}$ from the edge below the contact. The characteristic behavior for $\mathrm{Fe}$ and

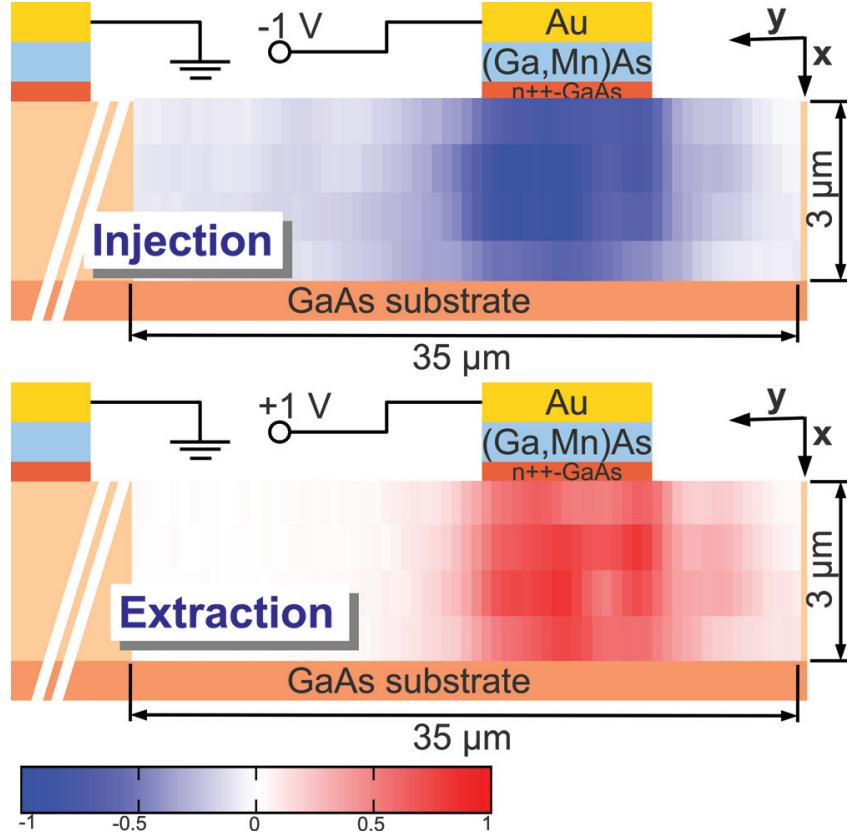

FIG. 2. (Color online) 2-dimensional scans of the Kerr rotation in the nGaAs channel, shown as a color coded map for spin injection and extraction from a $\mathrm{Ga}_{95} \mathrm{Mn}_{5}$ As contact.

(Ga,Mn)As injectors is practically the same for all contact lengths between 8 and $>100 \mu \mathrm{m}$.

The spatial spin density distribution for both contact materials can be qualitatively understood with the following assumptions:

1. The injecting contact represents an equipotential surface.

2. The voltage drop along the n-GaAs channel and the current density distribution are affected both by the channel resistance and the interface resistance.

For the $(\mathrm{Ga}, \mathrm{Mn})$ As sample, the interface resistance is Ohmic and much larger than the channel resistance. As a

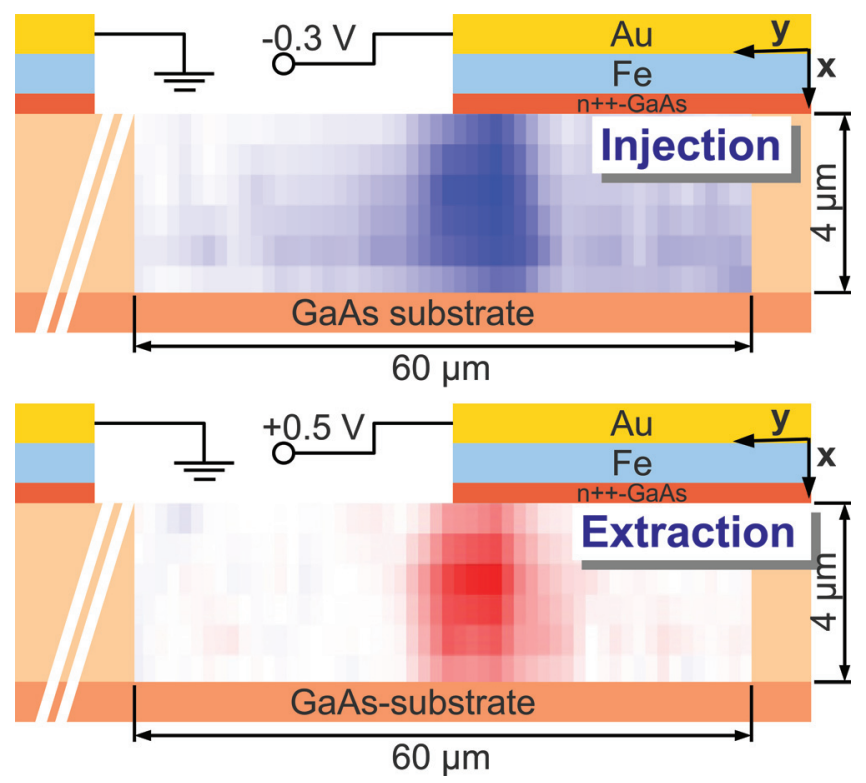

FIG. 3. (Color online) 2-dimensional scans of the Kerr rotation in the n-GaAs channel, shown as a color coded map for spin injection and extraction from an $\mathrm{Fe}$ contact. 

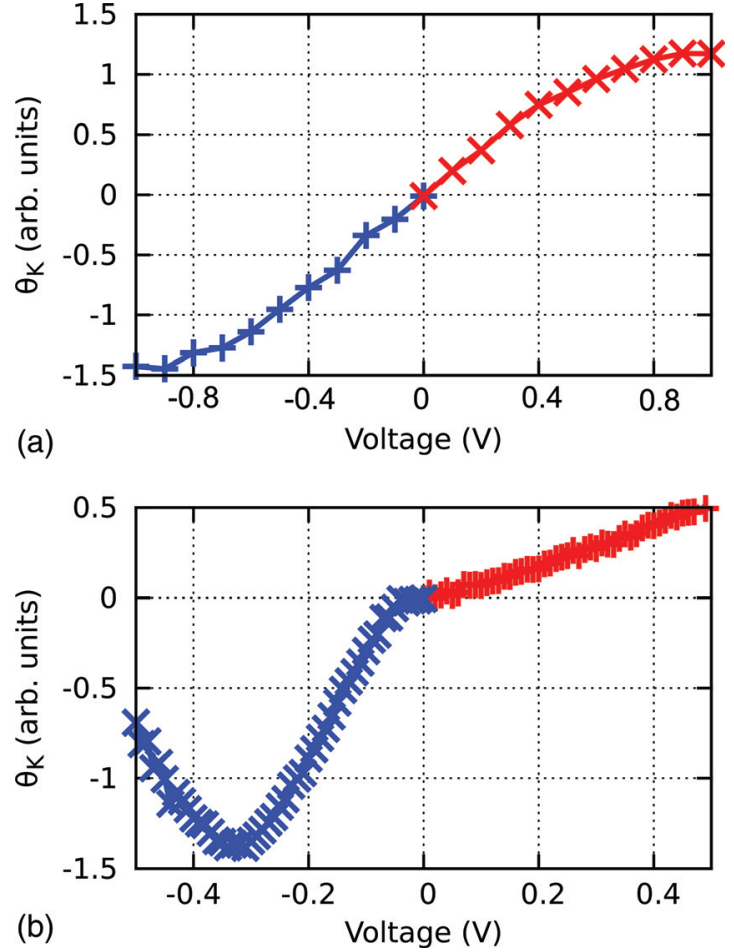

FIG. 4. (Color online) Comparison of the bias dependence of the injected spin polarization below the contact for $(\mathrm{Ga}, \mathrm{Mn}) \mathrm{As} / \mathrm{n}-\mathrm{GaAs}$ (a) and $\mathrm{Fe} / \mathrm{n}$ GaAs (b).

consequence, the current density, $\mathrm{j}_{x}$, across the interface is practically uniform along the channel. The current density along the channel, $\mathrm{j}_{y}$, increases from right to left below the contact. The spin polarization along the channel results from the interplay between current density and spin relaxation. Experimentally, this leads to a nearly uniform spin density below the entire contact with a weak tendency to an increase from right to left as seen in Fig. 2. In contrast, for Fe (or $\mathrm{FeCo}$ ) injectors $\mathrm{I}(\mathrm{V})$ curves between different contacts indicate that the interface resistance of the Schottky barrier for a bias voltage of $0.5 \mathrm{~V}$ is of the same order of magnitude as the channel resistance. Therefore, the voltage across the metal/GaAs interface decreases from left to right and, due to the nearly exponential $\mathrm{I}(\mathrm{V})$ characteristic of the Schottky contact, the current density and the spin polarization show a drastic decrease away from the left contact edge. A quantitative description of this effect is expected from numerical simulations of the two-dimensional potential and current density distribution within the sample structure.

The results shown in Figs. 2 and 3 are of considerable relevance for electrical measurements of the spin injection in a nonlocal geometry; e.g., in Hanle effect measurements, the assumed position of the injected spin polarization directly affects the extracted spin lifetime. Here the effective distance between the injecting and the sensing contact may be different from the geometric contact pattern and shift with the applied bias voltage. In such a case, a unique feature of our imaging technique is the possibility to directly evidence the actual position of the spin injection.

The bias dependence of the injected spin polarization is shown in Fig. 4 for a $(\mathrm{Ga}, \mathrm{Mn}) \mathrm{As}$ and an Fe contact. For $(\mathrm{Ga}, \mathrm{Mn})$ As the bias dependence was measured in the center below the contact while for the Fe sample it was measured at the edge. Both graphs clearly show a sign reversal when switching from injection to extraction.

In contrast to $\mathrm{Fe}$ and $\mathrm{FeCo}$ as injector materials the bias dependence for $(\mathrm{Ga}, \mathrm{Mn}) \mathrm{As}$ is almost symmetric. This is probably connected with the band structure of $(\mathrm{Ga}, \mathrm{Mn}) \mathrm{As}$ and GaAs and the band bending in the Esaki diode structure. ${ }^{4}$

Finally, the unexpected sign reversal of the spin polarization as a function of the bias voltage for $\mathrm{Fe}$ on GaAs reported in Ref. 1 for small bias voltages will be addressed. It was suggested that surface bands are created by disorder from which the preferred extracted spin is opposite to that from the bulk conduction band, ${ }^{5}$ or that the observed sign reversal results from an interface resonant band which strongly contributes to the tunneling conductance. ${ }^{6}$ The fact that no sign reversal of current spin polarization was seen by Kotissek et $a .^{2}$ for injection from a bcc epitaxial FeCo contact raised the question whether the different behavior was due to the larger band filling expected for bcc FeCo compared to bcc Fe. The present results for an Fe contact as seen in Fig. 4 show that a different band filling is not the main reason for the different behavior found in Refs. 1 and 2 . Instead, this indicates that the metal/GaAs interface resulting from the particular sample preparation conditions plays a decisive role. The strong influence of the interface between $\mathrm{Fe}$ and GaAs was recently confirmed by Schultz et al. by studying the effect of growth and annealing temperature on the sign of the injected spin polarization. ${ }^{7}$

Financial support by the Deutsche Forschungsgemeinschaft (SFB 689) is gratefully acknowledged.

${ }^{1}$ X. Lou, C. Adelmann, S. A. Crooker, E. S. Garlid, J. Zhang, K. S. M. Reddy, S. D. Flexner, C. J. Palmstrøm, and P. A. Crowell. Nat. Phys. 3, 197 (2007).

${ }^{2}$ P. Kotissek, M. Bailleul, M. Sperl, A. Spitzer, D. Schuh, W. Wegscheider, C. H. Back, and G. Bayreuther. Nat. Phys. 3, 872 (2007).

${ }^{3}$ M. Ciorga, A. Einwanger, U. Wurstbauer, D. Schuh, W. Wegscheider, and D. Weiss. Phys. Rev. B 79, 165321 (2009).

${ }^{4}$ P. Van Dorpe, W. Van Roy, J. De Boeck, G. Borghs, P. Sankowski, P. Kacman, J. A. Majewski, and T. Dietl. Phys. Rev. B 72, 205322 (2005).

${ }^{5}$ H. Dery and L. J. Sham. Phys. Rev. Lett. 98, 046602 (2007).

${ }^{6}$ Athanasios N. Chantis, Kirill D. Belashchenko, Darryl L. Smith, Evgeny Y. Tsymbal, Mark van Schilfgaarde, and Robert C. Albers. Phys. Rev. Lett. 99, 196603 (2007).

${ }^{7}$ B. D. Schultz, N. Marom, D. Naveh, X. Lou, C. Adelmann, J. Strand, P. A. Crowell, L. Kronik, and C. J. Palmstrøm. Phys. Rev. B 80, 201309 (2009). 